\title{
Impediments to Compliance with Hand Hygiene among Medical and Dental Students in Medical and Dental College
}

\author{
Harshita Hemant Sisodiya ${ }^{1}$, Sneha Hemant Sisodiya ${ }^{2}$, Nikhil Jaykumar Gandhi ${ }^{3}$, \\ Avinandan Shantanu Saha ${ }^{4}$, Madhav Sathe ${ }^{5}$, Puja Ambrish Iyengar ${ }^{6}$ \\ ${ }^{1}$ Assistant Professor Microbiology, TNMC \& BYL Nair Hospital, Mumbai \\ ${ }^{2}$ Assistant Professor Pathology, LTMGHMC \& Sion Hospital, Mumbai \\ ${ }^{3}$ Senior Resident, Orthopaedics, VN Desai Hospital, Santacruz. Mumbai \\ ${ }^{4}$ Senior Resident, Microbiology, Seth GSMC \& KEM Hospital, Mumbai \\ ${ }^{5}$ Professor Microbiology, TNMC \& BYL Nair Hospital, Mumbai \\ ${ }^{6}$ Consultant Pathologist, Bai Jerbai Wadia Hospital, Mumbai \\ Corresponding Author: Harshita Sisodiya
}

\begin{abstract}
Introduction: Hand hygiene is the first line of defense and a cost-effective method for reducing healthcare-associated infections (HCAIs) and antibiotic resistance. It is essential for healthcare professionals to have adequate knowledge regarding hand hygiene. Ensuring proper education of trainees is required as they are the healthcare providers of the future.

Aim: To assess the impediments that both medical and dental trainees face in performing effective hand hygiene.

Materials and Methods: The study questionnaire based on WHO's concepts of "Five Moments for Hand Hygiene" and "Six Steps of Hand Hygiene" was sent to 225 participants from $2^{\text {nd }} \& 3^{\text {rd }}$ MBBS and BDS courses. Associations between variables of interest were tested using the chi-square $\left(\chi^{2}\right)$ test. Statistical analysis was carried out using the Statistical Package for the Social Sciences version 17. The p-value $<0.05$ was considered statistically significant.

Results: Total 184 (81.9\%) students from medical and 41(18.2\%) from dental stream participated, 118 out of 225 were females and 107 were males.

Out of 225, $175(77.8 \%)$ participants used soap and water, 32 (14.2\%) used hand rub.

Also 98 out of 225 (43.6\%) participants think that because of workload the steps of handwashing were missed. The various facilities like access to water, wash basin, soap, hand-rub, facilities for drying hands required for hand hygiene may be lacking as per $31.6 \%$ participants.

It was noticed that 96 out of $225(42.7 \%)$ use hand rub for less than 10 seconds duration, $86(38.2 \%)$ for $11-20$ seconds, $30(13.3 \%)$ for $21-30$ seconds while only $13(5.8 \%)$ used hand-rub for than 30 seconds.

For performing handwash, 141 out of 225 (62.7\%) used cloth towel, 62(27.6\%) used paper-towel and $22(9.8 \%)$ used dryer for drying hands.

205 out of 225 (91.1\%) participants think increasing the frequency of training and audits will help in improving the hand hygiene.

Conclusion: Formal training in of hand hygiene needs to be incorporated in teaching at the undergraduate level which would ensure adequate and appropriate utilization of resources. Provision of adequate hand hygiene supplies, particularly soap and facilities for drying hands needs to be ensured.
\end{abstract}

Key Words: Healthcare associated infections, Hand rub, Hand wash, Undergraduate students 


\section{INTRODUCTION}

Pathogens are readily transmitted through the hands of Health Care Workers (HCWs). (1) Appropriate and adequate hand hygiene is the first line of defense against the transmission of infectious microorganisms both in the community and in healthcare settings, and a cost-effective method for reducing healthcare-associated infections (HCAIs) and preventing the spread of antimicrobial resistance.

Healthcare-associated infections are an important and expensive public health problem. It is estimated that approximately 1 out of every 20 hospitalized patients will contract an HCAI. (2) Hands have been identified as the major source of germ transmission while providing health care. (3) This risk is also to HCWs who may contract infectious diseases from patients. (4) Hence, it is essential for healthcare professionals to have adequate knowledge regarding hand hygiene practices to reduce the burden of these illnesses.

Despite several standards and guidelines on hand hygiene, compliance, especially among health care providers, is reported to be less than $40 \%$. (1) (5-6) Amongst several strategies to improve compliance, ensuring proper education of trainees is required as they are the healthcare providers of the future and their current patterns of training will reflect on their infection control practices later as qualified physicians.

Medical and dental students come into direct contact with patients during training. (6) Their knowledge, attitude, and performance in this period of clinical training are a cornerstone for their performance in the future. Despite the importance attributed to hand hygiene in literature, compliance among qualified physicians is abysmally low (average: 38.7\%). (7-8) However, effective teaching and the presence of a mentor during their trainee years has been shown to change attitudes toward hand hygiene practices and policies. (4)
Hand hygiene performance can be done by direct observation, measuring product use and conducting surveys.

It is important to investigate the reasons for non-adherence to hand hygiene to examine the organizational context of health care delivery, which may facilitate or inhibit adherence.

This observational study was carried out to assess the impediments that both medical and dental trainees face in performing effective hand hygiene. This will enable formulation and implementation of effective management policies and practices to counter these impediments which can then be perpetuated by this population of future clinicians.

\section{MATERIALS \& METHODS}

A sample size of 225 students was calculated considering a power of $90 \%$, an absolute precision of $5 \%$ and a confidence level of $95 \%$, assuming the awareness levels for hand hygiene to be $50 \%$ among medical and dental students. After obtaining Institutional and ethical approvals, the study included consenting participants from $2^{\text {nd }} \&$ $3^{\text {rd }}$ MBBS and BDS courses.

The study questionnaire based on WHO's concepts of "Five Moments for Hand Hygiene" and "Six Steps of Hand Hygiene" was sent to participants via Google sheets via social media.

\section{Statistical Analysis}

Associations between variables of interest were tested using the chi-square $\left(\chi^{2}\right)$ test. Statistical analysis was carried out using the Statistical Package for the Social Sciences version 17 (SPSS Inc., Chicago, IL, USA). The p-value $<0.05$ was considered statistically significant.

\section{RESULTS}

In this study, 118 out of 225 were females and 107 were males, $\mathrm{M}: \mathrm{F}=1.1: 1$. Total $184(81.9 \%)$ students from medical and 41(18.2\%) from dental stream participated in this study. 
It is found that the source of information about hand hygiene could be through various means. Majority of them have heard of it in their undergraduate course.

Table 1. Source of Information regarding Hand hygiene \begin{tabular}{|l|l|}
\hline Source of information & Total no. \\
\hline
\end{tabular}

\begin{tabular}{|l|l|}
\hline Source of information & Total no. \\
\hline Undergraduate course & 167 \\
\hline Media & 39 \\
\hline Training at work & 78 \\
\hline Information, posters or leaflets & 52 \\
\hline Peers/ Faculty & 81 \\
\hline
\end{tabular}

In this study, the participants knew various methods by which hand hygiene can be carried out like soap and water, hand rub, or both of them or any of them.
Table 2. Methods known for hand-hygiene

\begin{tabular}{|l|l|l|}
\hline Methods of hand-hygiene Used & Frequency & Percent \\
\hline Soap and water & 36 & 16.0 \\
\hline Hand rub only & 2 & .9 \\
\hline $\begin{array}{l}\text { Only 2 (Soap and Water) and 3 (Hand } \\
\text { rub only) }\end{array}$ & 152 & 67.6 \\
\hline Any of the above & 35 & 15.6 \\
\hline
\end{tabular}

Out of 225, $175(77.8 \%)$ participants most often use soap and water, 32 (14.2\%) used hand rub while 18 (8\%) used only water.

Table 3. Moments Missed

\begin{tabular}{|l|l|}
\hline \multicolumn{2}{|c|}{ Table 3. Moments Missed } \\
\hline Moments Missed & Total \\
\hline Before touching patients & 120 \\
\hline After touching patients & 27 \\
\hline Before performing procedure & 32 \\
\hline After performing procedure & 13 \\
\hline After leaving clinic & 96 \\
\hline Before meals & 32 \\
\hline
\end{tabular}

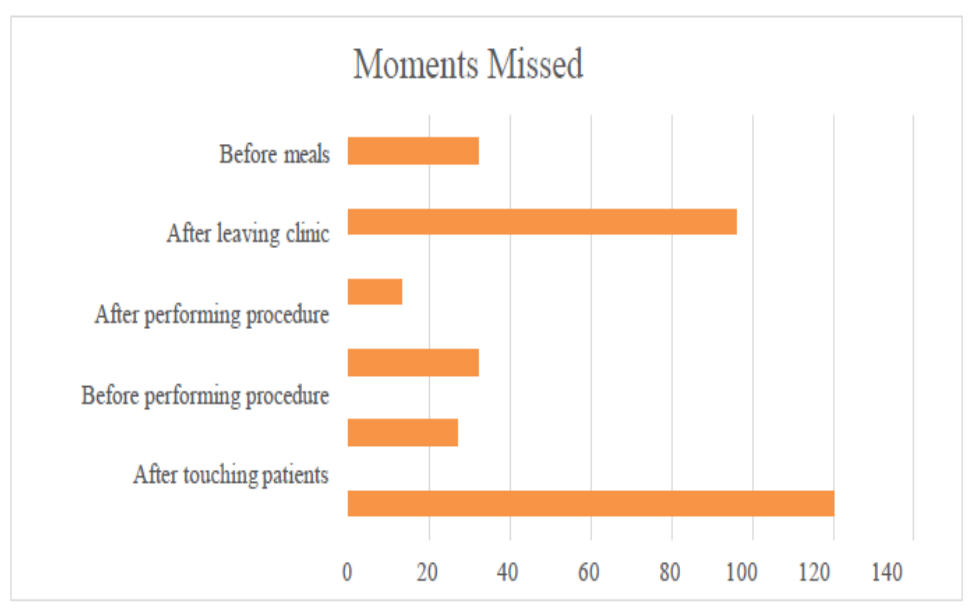

Figure 1. Moments missed for hand hygiene

Table 4. Duration for which hand washing is done

\begin{tabular}{|l|l|l|}
\hline \multicolumn{3}{|l|}{ When you wash your hands how long do you wash it for } \\
\hline & Frequency & Percent \\
\hline $11-20$ Seconds & 75 & 33.3 \\
\hline 21-30 Seconds & 61 & 27.1 \\
\hline 31-40 Seconds & 20 & 8.9 \\
\hline $41-50$ Seconds & 10 & 4.4 \\
\hline 51-60 Seconds & 15 & 6.7 \\
\hline Less than 10 seconds & 31 & 13.8 \\
\hline More than a minute & 13 & 5.8 \\
\hline
\end{tabular}

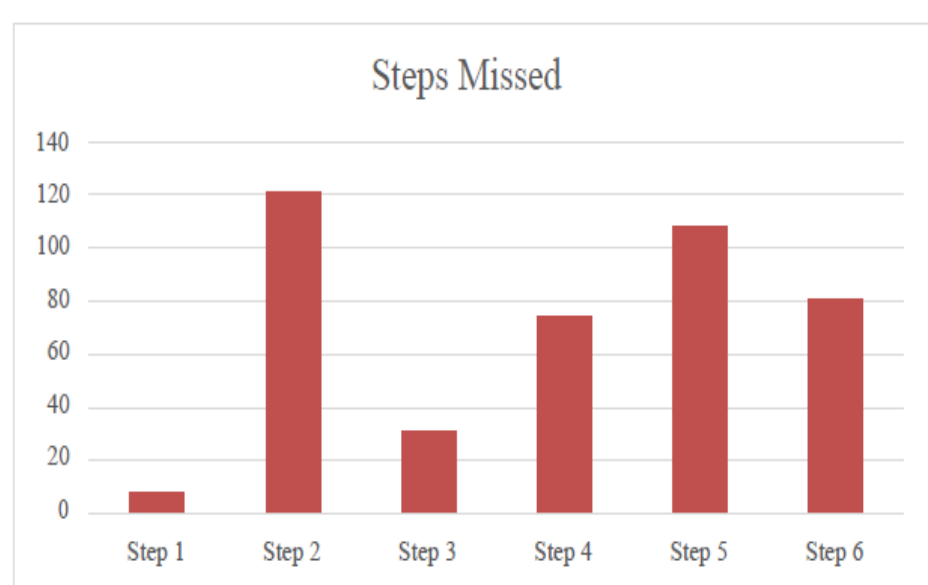

Figure 2. Steps missed during handwashing 
Also 98 out of $225 \quad(43.6 \%)$ participants think that because of workload the steps of handwashing were missed. The various facilities like access to water, wash basin, soap, hand-rub, facilities for drying hands required for hand hygiene may be lacking as per $31.6 \%$ participants of the study.

To compare medical and dental participants, in both the streams, excess workload is the factor resulting in missing moments or steps of hand hygiene.

It is noticed that 96 out of 225 $(42.7 \%)$ use hand rub for less than 10 seconds duration, $86(38.2 \%)$ for $11-20$ seconds, 30 (13.3\%) for 21-30 seconds while only $13(5.8 \%)$ used hand-rub for than 30 seconds.

So, there are total 33 from medical stream and 10 from dental using hand-rub for more than 20 seconds while 151 and 31 participants respectively from medical and dental field who use hand- rub for less than 20 seconds.

For performing handwash, 141 out of $225(62.7 \%)$ used cloth towel, 62(27.6\%) used paper-towel and 22(9.8\%) used dryer for drying hands.

206 out of $225(91.6 \%)$ participants think that setting aside time before rounds, between patients and after rounds will help in improving the hand hygiene \& others don't agree with this.

205 out of $225(91.1 \%)$ participants think increasing the frequency of training and audits will help in improving the hand hygiene others don't agree with this.

\section{DISCUSSION}

The present study evaluated knowledge, attitudes and practices regarding hand hygiene at a grassroots level among undergraduate medical and dental students recently entered into clinical training and to identify the impediments to effective hand hygiene practice faced by them.

Our study sample comprised predominantly medical undergraduate students from second and third years (82\%), and $18 \%$ were dental students from second and third years.

Nearly all study participants had come across the concept of hand hygiene before. Out of 225 medical \& dental students, 223 students were aware of the term from their undergraduate coursework, peers and faculty, and from training programmes at work.

Most students (68\%) were aware of the methods of hand hygiene, i.e., using soap and water or alcohol-based hand rub, and a majority (78\%) used soap and water.

There was no difference between medical and dental students with regard to their knowledge of hand hygiene steps. However, only $13 \%$ performed hand hygiene for the WHO recommended duration, with a majority (43\%) from both fields completing all six steps in less than 10 seconds with hand rub. There was no difference between medical and dental students in this regard. The steps missed most often were washing of the back of hands, fingertips and wrists. Similar results were reported by Sultana, et al. who observed that a majority washed their hands with soap and water but only $22.5 \%$ followed the correct steps and frequency of hand hygiene. (9)

Also, nearly half (44\%) admitted to missing hand hygiene indications and that they should be performing hand hygiene more frequently. The indications missed most often were before patient contact and after contact with patient environment. Al Kadi, et al. found that only $29 \%$ of students were able to identify all the five indications, and recorded a low compliance of $17 \%$. (10) In other studies, Van de Mortel et al. found that $63 \%$ of medical students were aware of the correct indications for hand hygiene; Mann and Wood found that only $56 \%$ of students were aware; and Graf et al. recorded an awareness of $33 \%$ among their students. (11-13)

Feather et al. studied the hand hygiene practices of 187 candidates found that only $8.5 \%$ of candidates washed their hands after patient contact. (14) However, 
compliance increased to $18.3 \%$ when instructive signs were displayed.

A majority (64\%) ascribed shortage of time as a reason for missing out on essential steps of hand hygiene, with workload being an important factor $(77 \%)$. A majority (92\%) felt setting aside time dedicated solely to hand hygiene before and after rounds and in between patients would help increase compliance. Most (91\%) also felt training sessions and audits would help increase compliance. Both medical and dental students expressed these same views.

Although lack of access to facilities was not a major impediment to hand hygiene compliance, provision of soap and facilities for drying hands were found to be wanting by most students. Availability of soap was also found to be an impediment to hand hygiene by Sultana, et al. (9)

Similarly, although various guidelines recommend the use of paper towels or dryers for drying hands, 141 $(62.7 \%)$ students from both medical and dental fields used a cloth towel. This was chiefly due to non-availability of the former drying facilities.

Researchers have found a number of factors for the lack of hand hygiene awareness and compliance in medical students.

The influence of role models and mentors has a significant impact on training. Frequent changes in role models with each passing year of training, along with practice of faulty hand hygiene by such role models, affects hand hygiene practice among students as mentioned by Lankford et al. (15)

The lack of enforcement of hand hygiene concepts and skills in the training syllabus can lead to a disregard for this vital concept by students. Anwar et al. reported that only $17 \%$ of interns and postgraduate medical students were aware of WHO recommendations on hand hygiene. (16) Mann and Wood et al. in 2006 examined the infection control knowledge of third year medical students at a UK medical school using a semi-structured questionnaire which included a hand hygiene component. (12) The mean hand hygiene knowledge score was $52.3 \%$, while $58 \%$ did not know the correct indications for the use of alcohol hand rub.

Hand hygiene compliance reflects the attitudes, behavior, and beliefs of healthcare personnel and the importance of developing correct hygiene behavior in the medical education years has been repeatedly stressed by Van de Mortel et al. \& Stone S. P. et al. (11) (17) Van de Mortel et al. compared the hand hygiene knowledge, beliefs, and practices between nursing and medical students and found that the nursing students had better knowledge and more positive beliefs of hand hygiene than medical students. (11) In our study, we found no difference in these between medical and dental students. Graf et al. in 2011 studied the attitudes and beliefs of medical students when they were being promoted from the basic to the clinical phase and noticed a major lack of information regarding the indications of proper hand hygiene. (13)

As in our study, the failure to translate awareness of hand hygiene into compliance is evident from other studies as well. (18)

Previous studies on dental students have identified a poor knowledge, low attitude, and behaviour towards hand hygiene and infection control. (19-21) However, there is insufficient literature that assesses this topic among both medical and dental students. We found that the knowledge, attitudes and practices of medical and dental students with regard to hand hygiene were deficient in the same aspects for each group, viz., indications of hand hygiene, duration for which hand hygiene should be performed and a lack of time.

Hand hygiene is the single most cost-effective method to prevent the spread of infections. Hand hygiene should become an educational priority. (14) Evidence-based standards by WHO and Centers for Disease Control and Prevention serve as a standard 
against which to measure current knowledge, attitudes and practices around this vital concept. (8)

This study was conducted in a single institute. It was a cross-sectional study with a limited sample size that used a selfreporting questionnaire for assessment.

\section{CONCLUSION}

Our findings suggest that formal training in hand hygiene needs to be incorporated in teaching and assessment at the undergraduate level for both medical and dental courses alike. Of particular importance is training in the indications of hand hygiene, steps of hand hygiene and indications for hand wash versus hand rub. This would ensure adequate and appropriate utilization of resources. Provision of adequate hand hygiene supplies, particularly soap and facilities for drying hands needs to be ensured.

\section{Acknowledgement: None}

\section{Conflict of Interest: None}

\section{Source of Funding: None}

\section{REFERENCES}

1. Allegranzi B, Pittet D. Role of hand hygiene in healthcare-associated infection prevention. Journal of hospital infection. 2009 Dec 1;73(4):305-15.

2. Kulkarni V, Papanna MK, Mohanty U, Ranjan R, Neelima V, Kumar N, Mithra PP, Upadhyay RP, Unnikrishnan B. Awareness of medical students in a medical college in Mangalore, Karnataka, India concerning infection prevention practices. Journal of infection and public health. 2013 Aug 1;6(4):261-8.

3. Modi PD, Kumar P, Solanki R, Modi J, Chandramani $S$, Gill N. Hand hygiene practices among Indian medical undergraduates: a questionnaire-based survey. Cureus. 2017 Jul;9(7).

4. Ibrahim AA, Elshafie SS. Knowledge, awareness, and attitude regarding infection prevention and control among medical students: a call for educational intervention.
Advances in medical education and practice. 2016;7:505.

5. Pittet D, Allegranzi B, Boyce J, World Health Organization World Alliance for Patient Safety First Global Patient Safety Challenge Core Group of Experts. The World Health Organization guidelines on hand hygiene in health care and their consensus recommendations. Infection Control \& Hospital Epidemiology. 2009 Jul;30(7):611-22.

6. Hughes RG, editor. Patient Safety and Quality: An Evidence-Based Handbook for Nurses. Rockville (MD): Agency for Healthcare Research and Quality (US); 2008 Apr. PMID: 21328752.

7. Karaaslan A, Kepenekli Kadayifci E, Atıc1 S, Sili U, Soysal A, Çulha G, Pekru Y, Bakır M. Compliance of healthcare workers with hand hygiene practices in neonatal and pediatric intensive care units: overt observation. Interdisciplinary perspectives on infectious diseases. 2014 Jan 1;2014.

8. Boyce JM, Pittet D. Guideline for hand hygiene in health-care settings: recommendations of the Healthcare Infection Control Practices Advisory Committee and the HICPAC/SHEA/APIC/IDSA Hand Hygiene Task Force. Infection Control \& Hospital Epidemiology. 2002 Dec;23(S12):S3-40.

9. Sultana M, Mahumud RA, Sarker AR, Hossain SM. Hand hygiene knowledge and practice among university students: evidence from private universities of Bangladesh. Risk management and healthcare policy. 2016;9:13.

10. Al Kadi A, Salati SA. Hand hygiene practices among medical students. Interdisciplinary perspectives on infectious diseases. 2012 Sep 16;2012.

11. Van de Mortel TF, Apostolopoulou E, Petrikkos G. A comparison of the hand hygiene knowledge, beliefs, and practices of Greek nursing and medical students. American journal of infection control. 2010 Feb 1;38(1):75.

12. Mann CM, Wood A. How much do medical students know about infection control?. Journal of Hospital Infection. 2006 Dec 1;64(4):366-70.

13. Graf K, Chaberny IF, Vonberg RP. Beliefs about hand hygiene: a survey in medical students in their first clinical year. American 
journal of infection control. 2011 Dec 1;39(10):885-8.

14. Feather A, Stone SP, Wessier A, Boursicot KA, Pratt C. 'Now please wash your hands': the handwashing behaviour of final MBBS candidates. Journal of Hospital Infection. 2000 May 1;45(1):62-4.

15. Lankford MG, Zembower TR, Trick WE, Hacek DM, Noskin GA, Peterson LR. Influence of role models and hospital design on the hand hygiene of health-care workers. Emerging infectious diseases. 2003 Feb;9(2):217.

16. Anwar MA, Rabbi S, Masroor M, Majeed F, Andrades M, Baqi S. Self-reported practices of hand hygiene among the trainees of a teaching hospital in a resource limited country. JPMA. The Journal of the Pakistan Medical Association. 2009 Sep 1;59(9):631.

17. Stone SP. Hand hygiene- the case for evidence-based education. Journal of the Royal Society of Medicine. 2001 Jun;94(6):278-81.

18. Han K, Dou FM, Zhang LJ, Zhu BP. Compliance on hand-hygiene among healthcare providers working at secondary and tertiary general hospitals in Chengdu. Zhonghua liu xing bing xue za zhi=
Zhonghua liuxingbingxue zazhi. 2011 Nov 1;32(11):1139-42.

19. Thivichon-Prince B, Barsotti O, Girard R, Morrier JJ. Hand hygiene practices in a dental teaching center: Measures and improve. European journal of dentistry. 2014 Oct;8(04):481-6.

20. Abreu MH, Lopes-Terra MC, Braz LF, Rímulo AL, Paiva SM, Pordeus IA. Attitudes and behavior of dental students concerning infection control rules: a study with a10-year interval. Brazilian dental journal. 2009;20(3):221-5.

21. Myers R, Larson E, Cheng B, Schwartz A, Da Silva K, Kunzel C. Hand hygiene among general practice dentists: a survey of knowledge, attitudes and practices. The Journal of the American Dental Association. 2008 Jul 1;139(7):948-57.

How to cite this article: Sisodiya HH, Sisodiya SH, Gandhi NJ et.al. Impediments to compliance with hand hygiene among medical and dental students in medical and dental college. Int J Health Sci Res. 2021; 11(10): 260266. DOI: https://doi.org/10.52403/ijhsr. 20211034 